\title{
Review Article \\ DTI Measurements in Multiple Sclerosis: Evaluation of Brain Damage and Clinical Implications
}

\author{
Emilia Sbardella, Francesca Tona, Nikolaos Petsas, and Patrizia Pantano \\ Department of Neurology and Psychiatry, Sapienza University, Viale dell'Università 30, 00185 Rome, Italy \\ Correspondence should be addressed to Emilia Sbardella; emilia.sbardella@uniromal.it
}

Received 23 December 2012; Revised 20 February 2013; Accepted 5 March 2013

Academic Editor: Rob Bermel

Copyright ( 2013 Emilia Sbardella et al. This is an open access article distributed under the Creative Commons Attribution License, which permits unrestricted use, distribution, and reproduction in any medium, provided the original work is properly cited.

\begin{abstract}
Diffusion tensor imaging (DTI) is an effective means of quantifying parameters of demyelination and axonal loss. The application of DTI in Multiple Sclerosis (MS) has yielded noteworthy results. DTI abnormalities, which are already detectable in patients with clinically isolated syndrome (CIS), become more pronounced as disease duration and neurological impairment increase. The assessment of the microstructural alterations of white and grey matter in MS may shed light on mechanisms responsible for irreversible disability accumulation. In this paper, we examine the DTI analysis methods, the results obtained in the various tissues of the central nervous system, and correlations with clinical features and other MRI parameters. The adoption of DTI metrics to assess the outcome of prognostic measures may represent an extremely important step forward in the MS research field.
\end{abstract}

\section{Introduction}

In multiple sclerosis (MS) research, nonconventional magnetic resonance imaging (MRI) techniques have demonstrated a high degree of specificity and sensitivity in detecting pathological tissue damage [1]. These techniques include diffusion-weighted imaging, which plays an important role in highlighting brain microstructural damage not visible when conventional sequences are used. Diffusion imaging principles are based on the measurement of motion of water molecules within tissues [2]. Free water usually moves equally in all directions in an isotropic fashion; when, however, water is restricted inside or by tissues, preferential directions are taken and movement consequently becomes anisotropic. Therefore, water mobility in the brain is markedly reduced in compact tissue, such as white matter (WM), is reduced to a lesser extent in the grey matter (GM), and is almost free in the cerebrospinal fluid (CSF). Pathological processes that alter the normal brain structure may affect water motion, with effects on the resulting diffusion indexes.

Diffusion images can be acquired from a minimum of three gradient directions, which yield two different kinds of sequences: diffusion-weighted imaging (DWI) and diffusion tensor imaging (DTI), respectively. The diffusion tensor is a matrix acquired from at least 6 gradient directions that characterizes three-dimensional water movement. It can be represented as an ellipsoid whose components are 3 main axes [3] (Figure 1): the longest axis stands for the primary eigenvector $\left(\lambda_{1}\right)$ and reflects diffusion parallel to the fibers, or axial diffusivity (AD); the two shorter axes represent the second $\left(\lambda_{2}\right)$ and third $\left(\lambda_{3}\right)$ eigenvectors and are averaged to provide a measure of diffusivity perpendicular to the fibers, or radial diffusivity (RD) [4]. The aforementioned metrics have proved to be able to discriminate between axonal damage and demyelinated damage: the former is better expressed by $\mathrm{AD}$, a measure of axonal integrity, and the latter by $\mathrm{RD}$, a measure of myelin integrity [5-8] (Table 1). Indeed, the diffusion study of shiverer mice, which have incomplete myelin formation in the CNS not accompanied by inflammation or axonal injury processes, helped to better define the meaning of $\mathrm{AD}$ and $\mathrm{RD}$ [5]. The authors of that study demonstrated that RD was significantly higher in shiverer mice than in controls, whilst $\mathrm{AD}$ was unchanged, thus pointing to $\mathrm{RD}$ and $\mathrm{AD}$ as markers of myelin and axonal integrity, respectively.

In WM tracts of MS patients, RD is typically increased owing to the loss of myelin, whereas $\mathrm{AD}$ has been reported to be either increased or decreased in comparison with healthy subjects (HS) [6]. A decrease in $\mathrm{AD}$ may be the consequence 
TABLE 1: Schematic description of the main DTI parameters.

\begin{tabular}{|c|c|c|c|}
\hline & Unit of measure & Formula & Object measured \\
\hline FA & $\begin{array}{l}\text { Scalar value ranging } \\
\text { between } 0-1\end{array}$ & $\sqrt{\frac{1}{2}} \frac{\sqrt{\left(\lambda_{1}-\lambda_{2}\right)^{2}+\left(\lambda_{2}-\lambda_{3}\right)^{2}+\left(\lambda_{3}-\lambda_{1}\right)^{2}}}{\sqrt{\lambda_{1}^{2}+\lambda_{2}^{2}+\lambda_{3}^{2}}}$ & Fibers directionality/axonal loss \\
\hline MD & $\mathrm{mm}^{2} / \mathrm{sec}$ & $\left(\lambda_{1}+\lambda_{2}+\lambda_{3}\right) / 3$ & Amount of water diffusion/myelin loss \\
\hline $\mathrm{AD}$ & $\mathrm{mm}^{2} / \mathrm{sec}$ & $\lambda_{1}$ & $\begin{array}{l}\text { Diffusivity parallel to the fibers/myelin } \\
\text { and axonal content }\end{array}$ \\
\hline $\mathrm{RD}$ & $\mathrm{mm}^{2} / \mathrm{sec}$ & $\left(\lambda_{2}+\lambda_{3}\right) / 2$ & $\begin{array}{l}\text { Diffusivity perpendicular to the } \\
\text { fibers/myelin content }\end{array}$ \\
\hline
\end{tabular}

FA: fractional anisotropy; MD: mean diffusivity; AD: axial diffusivity; RD: radial diffusivity; mm: millimeters; sec: second.

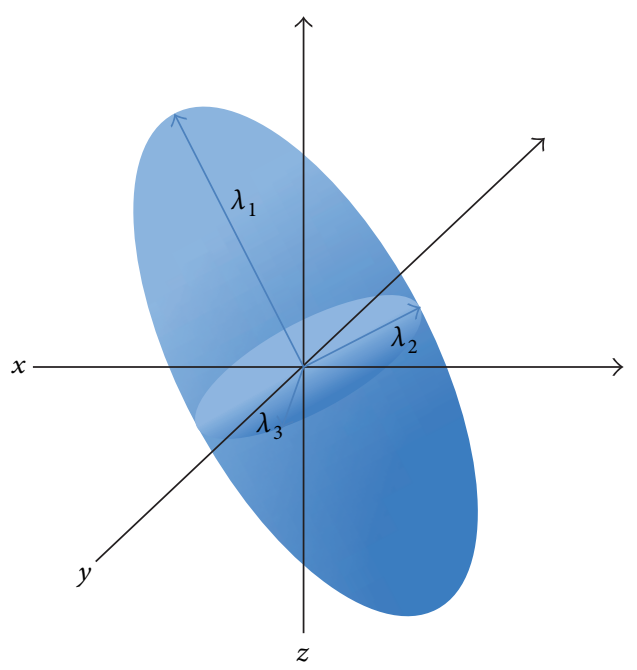

FIGURE 1: Elliptical representation of a diffusion tensor with the 3 main axes: the longest axis stands for the primary eigenvector $\left(\lambda_{1}\right)$, reflecting the diffusion parallel to the fibers; the two shorter axes represent the second $\left(\lambda_{2}\right)$ and third $\left(\lambda_{3}\right)$ eigenvectors, whose average provides a measure of diffusivity perpendicular to the fibers.

of axonal loss, whereas an increase has been interpreted as an attempt made by a compensative mechanism to maintain functionality in the presence of WM damage [6].

The DTI metrics used most, derived from a mathematical combination of the three eigenvectors [7], are mean diffusivity (MD), which measures overall water motion without any directionality, and fractional anisotropy (FA), which reflects the prevalence of diffusivity along one direction [8]. MD is a quantitative metric of water diffusion; the higher the MD value, the higher the diffusivity. FA is a scalar value ranging from 0 to 1 that is highest in compact WM tracts, decreases in the GM, and approaches zero in the CSF [8] (Table 1). In early studies, anisotropy was correlated to axon density and myelin content, while diffusivity was correlated above all to the amount of myelin [9]. However, both MD and FA have more recently been shown to be affected mainly by myelin content [10] and, to a lesser extent, by axonal density [11]. Briefly, MD has so far been interpreted as an index that is primarily influenced by free space, which means that processes such as vasogenic edema, axonal, and myelin loss increase its value [12]. On the other hand, FA is believed to be more sensitive to the detection of the integrity of WM, as significant FA differences have been observed between myelinated and nonmyelinated nerves [13-16]. Nonetheless, FA is not very specific and does not distinguish between diseases characterized by a range of pathological processes, such as edema, inflammation, demyelination, and leukoaraiosis [17].

The idea of diffusion MRI was introduced in 1986 [2] and was subsequently applied to the study of a number of neurological diseases, including MS [18]. Since it was first applied, methods of diffusion analyses have been steadily improved. Although region of interest (ROI) analysis was adopted in early MS studies [12, 14, 18-21], this method proved to have some drawbacks; that is, it is time-consuming, operator dependent, and subject to partial volume artifacts; furthermore, it does not provide a global assessment of tissue damage [22]. Accordingly, histogram analyses were carried out to evaluate the diffusion metrics in the whole brain [22-24]. Subsequently, a color was attributed to diffusion direction along each of the three planes of space, thus providing information on the direction of WM tracts [25].

Finally, in order to respond to a growing interest in the identification of regional diffusion changes, a whole brain voxel-based morphometry (VBM) approach [26, 27], usually applied to T1-weighted images to study atrophy, was used for diffusion images. However, owing to the limitations of this technique in terms of alignment inaccuracies and smoothing extent, VBM was partially superseded by tract-based spatial statistics (TBSS), a fully automated, whole brain diffusion analysis method [28].

Another way of studying diffusion images is based on the reconstruction of large fiber bundles using three-dimensional tractography [29-32] (Figure 2). This method delimits major tracts of WM in vivo: after the selection of one, or more than one, seed ROI, nervous pathways are reconstructed by tracking along the principal direction of the fibers passing through the ROI [33]. This technique can be used to analyze the displacement of fibers as well as to detect Wallerian degeneration [34]. It does, however, have some limitations, that is, difficulties to find the principal direction when there are many crossing fibers, low signal-to-noise ratio, poor spatial resolution, nondetectable small fibers, and difficulties in case of distorted brain, all of which require an a priori knowledge of anatomical structures [35]. To partially 


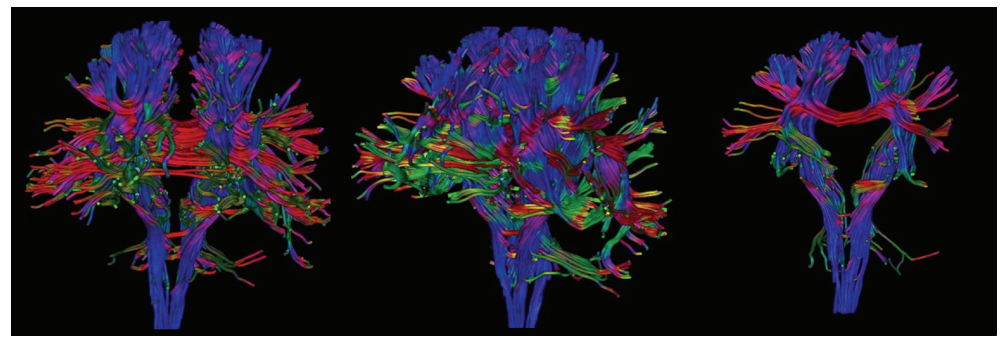

FIGURE 2: Three-dimensional tractography that reconstructs brain white matter bundles. The different colors represent different directions of the fibers.

overcome these problems, the use of more sophisticated mathematical models [36], constraints on the fiber tracking [37-39], and use of WM probability maps from HS [40] have been proposed.

\section{Diffusion Studies in Different MS Tissues}

Since diffusion MRI was first introduced, many works have applied DWI, and particularly DTI, to study patients affected by MS. Various methodological approaches of DTI analyses have been used to characterize different types of tissue damage. In this section, we describe the DTI results obtained in different types of brain and spinal cord tissues.

2.1. Normal Appearing White Matter (NAWM). In MS patients, widespread DTI abnormalities, consisting of increased MD and decreased FA, have been detected in NAWM $[1,12,19,23,41-44]$. A FA change gradient has also been demonstrated, with lower values being observed close to the plaques and higher values far from the plaques [44]. Histological studies, which have detected demyelination and axon transection even in WM outside the plaques $[10,45]$, suggest that DTI changes in NAWM may be ascribed to Wallerian degeneration processes [46].

Abnormalities in NAWM have been detected in almost all MS phenotypes, though the degree of damage varies according to the severity of the pathology. Indeed, although altered NAWM is visible from the onset of clinical symptoms, even at an early age, DTI-detectable damage becomes increasingly evident as the disease worsens.

Disability is less pronounced and pathological tissue damage is less severe in children with MS and in patients with clinically isolated syndrome (CIS) than in other phenotypes. In pediatric patients with CIS, at the very onset of disease, the NAWM diffusion metrics appear to be normal if compared with HS [47]. By contrast, increased RD and MD [27, 48, 49] and decreased FA and $\mathrm{AD}$ [50] values are found in most WM tracts in pediatric patients with definite MS and in adult patients with CIS compared with HS [47, 50-54]. Although agreement amongst researchers is not unanimous [27, 40, 55], these changes have been interpreted as a sign of early fiber loss in WM. Inconsistencies exist with regard to FA results, as some studies did not detect any changes. This may be explained by the fact that axonal transaction may, despite being present from disease onset [56], affect patients in different ways in the early stages and escape detection by DTI in the very early phases. By contrast, as the pathology progresses, the structural damage becomes more pronounced. Indeed, changes in NAWM are less evident in CIS than in patients with definite MS [27, 57]. Likewise, in patients with benign MS, FA and $\mathrm{AD}$ have been shown to be higher than those in relapsing remitting (RR) MS patients [27], although alterations in other DTI indexes, that is, MD and RD [58], have not been detected. This discrepancy points to the existence of neuroprotective mechanisms that may prevent axonal injury $[27,59]$ and clinical worsening, despite the inflammatory process. Moreover, DTI metrics have revealed differences between benign and RRMS patients in the topographical distribution of WM damage, which might be associated with the favorable clinical status in the former group [59].

Most [27], though not all $[24,60]$, studies, have detected differences in NAWM diffusion metrics between RRMS patients and HS. The lack of changes in RRMS observed in some studies is likely to be attributable either to the fact that subtle damage is not easily detected in the early phase of the disease or to the poor sensitivity of the ROI methodology adopted in those studies.

In comparison with CIS, RR, and benign MS, secondary progressive (SP) MS patients exhibit more pronounced WM diffusion abnormalities [27, 61, 62]. The greater increase in diffusivity in SPMS than in other phenotypes may represent a more advanced phase of the disease, presumably characterized by a combination of axonal loss and tissue destruction processes with inflammatory events [62]. The high degree of axonal degeneration in such patients is confirmed by the widespread decrease in FA, not only within lesions but also in NAWM [27].

Although widespread diffusivity changes, consisting of increased $\mathrm{MD}, \mathrm{RD}$, and $\mathrm{AD}$ and decreased $\mathrm{FA}$, have been demonstrated in primary progressive (PP) MS if compared with HS [27, 63], NAWM is affected to a lesser degree in PPMS patients than in those with SPMS [27, 64], probably owing to the more pronounced inflammation present in the SP than in the PP phenotype.

2.2. Normal Appearing Grey Matter (NAGM). Besides involving the WM, MS also affects the GM, with microscopic 
damage being detectable even in the absence of macroscopic lesions $[65,66]$. Anatomical changes are usually visible in the deep [67-69] and cortical GM $[69,70]$ in most MS phenotypes [64, 71-73]. However, some studies using ROI analysis did not provide conclusive evidence regarding the presence of NAGM abnormalities $[60,72]$, which suggests that different analysis methods might yield contrasting results.

The fact that no DTI changes have been detected in the NAGM of early onset patients [51] indicates that the GM might be preserved at an early age. However, the pathological involvement of NAGM was demonstrated when adult patients with CIS were studied [54, 57], with increased MD and decreased FA being detected in such patients, though to a less severe degree than in RRMS patients $[54,57]$.

Adults with benign MS have also exhibited changes in DTI $[19,21,74]$, consisting in particular of an increase in MD [75], which thus suggests that demyelination prevails over axonal damage when the disease is clinically less severe.

Most recent works have detected abnormalities, consisting of both FA and $\mathrm{MD}[42,76]$ changes, in the cortical or subcortical NAGM of RRMS patients compared with controls [27]. While MD usually increases, FA has been found to either increase [42,77] or decrease [76]. This discrepancy may be explained by the phase of GM inflammation at the time of the analysis, with the prevailing activation in GM of microglia or inflammatory processes leading, respectively, to an increased or decreased FA [78]. Other authors have instead reported no diffusion abnormalities in patients with early MS when compared with controls $[1,60,77]$. The reasons for this discrepancy may be the use of ROIs, the improvement in processing steps, and the heterogeneity of the sample size.

SPMS patients display more pronounced GM diffusion abnormalities [27, 54, 64, 71], at both the deep and cortical levels [69], than other MS phenotypes, probably owing to the concomitant presence of a high degree of inflammation and degeneration in this group of patients.

2.3. Spinal Cord (SC). It has been demonstrated that NAWM and T2 lesions within the SC yield increased $\mathrm{AD}$ and $\mathrm{RD}$ and decreased FA if compared with normal tissues. These abnormalities have been correlated with the degree of demyelination [79], while FA has also been closely correlated with axonal density [9]. Given the high sensitivity displayed by $\mathrm{RD}$ to discriminate myelin content, a recent study proposed increased $\mathrm{RD}$ as a marker of increasing severity of demyelination [80].

When cervical cord was assessed in benign MS patients, few abnormalities, consisting of increased MD, were detected outside focal macroscopic lesions [81]. Similarly, patients with early onset MS exhibited a slight increase in the MD of the NAWM, which points to a mild degree of damage and explains the more favorable clinical course in early onset than in adult onset patients [82].

Conversely, reduced FA and increased MD were observed in NAWM and in surrounding demyelinating plaques [81, 8386] in RRMS patients as well as in the NAWM of patients without any lesions within the whole SC [87]. This finding points to widespread pathological involvement of the spine, regardless of the presence of T2 lesions. Subjects with a progressive phenotype, that is, both $\mathrm{PP}$ and SP patients, also displayed increased MD and decreased FA in the NAWM of cervical SC $[81,88]$.

2.4. Lesion Tissue. FA within T2 lesions is usually decreased and MD increased $[12,18-21,23,89]$. An overlap between MD and FA maps and T2 lesion distribution has been demonstrated in most MS phenotypes [27], with one exception being PPMS patients, in whom a discrepancy between regional WM diffusivity changes and T2-visible focal lesions has been found [69]. The absence of any overlap in PPMS between FA maps and T2 lesions [27] as well as of any correlation between diffusion metrics and lesion volume [90] lends support to the hypothesis that axonal damage and T2 lesions in this phenotype are, unlike those in other phenotypes, partially independent.

Pronounced FA and MD changes expressing both tissue damage and vasogenic edema have been detected in gadolinium-enhancing lesions (GEL) [24, 41, 89, 91, 92]. However, FA is more sensitive to pathological damage than MD [14, 62]. Indeed, in active lesions, FA values decrease according to the severity of tissue disruption [41], whilst MD values may increase, decrease, or be similar to those detected in chronic lesions $[12,19,41,89]$. Acute demyelinating lesions exhibiting restricted diffusion, which have been described recently, are thought to be caused by the presence of inflammatory infiltrate or cytotoxic edema involving oligodendrocytes [93].

T1 hypointense lesions (black holes) [94], characterized by severe tissue injury, are associated with the most severe diffusion alterations because of their pathological characteristics $[1,12,19,41,62,89]$.

Since lesions have also been detected in GM, diffusion parameters have been investigated in this structure as well [95]. FA in GM lesions has, unlike that in WM, been found to increase. This finding may be due to the different histological characteristics of GM focal lesions, which present a higher level of activated microglia and greater loss of dendrites and axons along with a lower degree of inflammation than WM lesions [78, 96, 97].

\section{DTI Metrics and Clinical Disability}

Nonconventional techniques may detect microstructural changes and correlate with the clinical impairment more closely than usual MRI measures (i.e., T2 lesions, GEL, and T1 hypointensities), thus partially overcoming the so-called "clinical-radiological paradox" [98].

Diffusion abnormalities are more pronounced in patients with a long disease duration and severe neurological disability $[54,64,71,99]$, accurately reflecting the clinical condition. Attempts to correlate DTI metrics of the brain and SC with the most widely used clinical disability scale, that is, the Expanded Disability Status Scale (EDSS) [100], have yielded controversial results, with some studies finding a significant correlation $[81,101-104]$ not detected by others $[6,60]$. The weak correlation between the EDSS and diffusion parameters 
may be due to several reasons: first of all, DTI measures, particularly when applied to NAWM, indicate pathologically affected, though still functioning, fiber tracts [6]; secondly, the EDSS is a global clinical index that is affected above all by the motor system and might consequently not be severely altered in the early disease stage; thirdly, when diffusion changes are evaluated in the whole brain, a clinical correlation with measures of disability is less likely to be found. Indeed, when diffusion was analyzed at the regional level, in specific tracts, more pronounced correlations with clinical features emerged; that is, the EDSS was correlated with DTI changes in motor tracts $[105,106]$ and with quantitative fiber tractography results [107].

Moreover, when correlations between regional DTI measures and clinical scales assessing specific clinical features were investigated, the role of microstructural damage in disability became more evident [108, 109]. In particular, oculomotor function impairment was associated with a focal DTI alteration in small brainstem fiber pathways [3]. In addition, diffusion abnormalities in the optic nerves were also significantly correlated with visual evoked potential parameters, suggesting that DTI metrics may be used as a surrogate measure of axonal damage $[110,111]$. Diffusion measures in the optic nerve, optic radiation, and regional brain areas related to visual cortices were also associated with visual acuity $[112,113]$ and retinal nerve fiber layer thickness [113].

Regional diffusivity studies have also been used to find the anatomical substrates underlying the impairment of other functional systems. Tractography studies have demonstrated a correlation between MD and FA alterations in specific WM tracts, such as the corticospinal tract (CST) and the Corpus Callosum (CC), and motor disability [114-117]. Furthermore, cerebellar DTI abnormalities have been correlated with upper and lower limb disability [118]. Similarly, altered DTI parameters along the cerebellar connections and supratentorial associative WM bundles were correlated with balance impairment [119].

DTI can also reveal tract injury responsible for cognitive dysfunction in MS patients [71, 72, 108, 120, 121]. Focal abnormalities, particularly in the CC, have been related to calculation, sequence learning, and memory [121-126]. Moreover, several studies have detected correlations between cognitive impairment and diffusion metrics abnormalities in the posterior thalamic radiations [121] as well as frontosubcortical fiber tracts [127] and the thalamus [77].

FA abnormalities in specific NAWM tracts have even been found to be significantly correlated with various cognitive abilities in pediatric patients in the early phase of disease [128].

Lastly, a strong correlation was found between DTI measures in the SC and disability, thus corroborating the role played by the pathological involvement of the spine in the clinical manifestations of the disease [86].

\section{DTI Metrics and Nonconventional MRI}

Diffusion studies have also been combined with other nonconventional MRI techniques, such as spectroscopic, magnetization transfer ratio (MTR), and functional MRI.
When MRI spectroscopy was applied together with DTI, the results were inconsistent. One early work did not find any correlation between DTI metrics and N-acetyl aspartate (NAA) values [129], thus suggesting that chronic metabolic dysfunction contributes to axonal pathology in MS. This result was not, however, confirmed in a more recent study, which reported correlations between diffusion metrics and NAA/Creatine ratios, thereby pointing to a link between microstructural and metabolic alterations [130]. This discrepancy is likely due to the fact that diffusion measures highlight changes induced by structural axonal loss, whereas NAA changes may be related to other, even transient, factors such as the functionality of neurons [129].

A multiparametric study, based also on MTR MRI, detected metabolic and diffusivity changes not related to MTR measures in the CC of CIS patients [131]. The widely reported lack of any correlation between diffusion metrics and MTR values [23, 44, 132, 133] suggests that these two methods may be sensitive to different pathological processes and may provide independent measures of damage [132].

Lastly, DTI has been used to quantify brain damage in functional MRI (fMRI) studies to investigate correlations between structural damage and functional changes. The results of most task-related fMRI studies support the idea that compensatory neuroplasticity is designed to maintain normal function in the presence of widespread microstructural damage. Indeed, altered DTI parameters have been found to correlate with an increase in $\mathrm{PMRI}$ activation during various tasks in MS patients [134-136]. In addition, the ultrastructural damage of specific WM tracts may affect cortical activity. For example, Lenzi et al. [136] found that MD values in the body of CC correlated with activation of the ipsilateral motor cortex during hand movements, thereby suggesting that functional changes in this area are related to the loss of transcallosal inhibitory fibres in MS [136]. More recent studies have correlated measures of anatomical and functional connectivity: in MS, distinct functional networks exhibit increases in functional connectivity despite widespread diffusivity abnormalities within WM [137].

DTI was used to investigate the relationship between WM damage and GM atrophy in CIS. Henry et al. (2009) measured diffusivity indexes in the thalamocortical tracts that connect WM lesions and the thalami. They found that both lesions and DTI values in thalamocortical tracts correlated with atrophy, which points to a direct relationship between WM lesions and thalamic atrophy [49]. By contrast, DTI metrics alterations in GM were not related to concomitant brain atrophy progression $[138,139]$.

\section{Future Directions}

Given their marked sensitivity in detecting structural tissue abnormalities in MS, DTI metrics have been used to monitor structural changes that occur during the course of this disease $[73,99,140]$ in both WM and GM. The potential of DTI parameters as prognostic markers of disease evolution has also been evaluated. Unfortunately, the results are not conclusive, probably because of differences in the methods used to conduct these investigations. 
Although abnormalities in RRMS patients were found throughout the brain, no longitudinal diffusion changes were observed in the follow-up [141], nor were any longitudinal FA changes observed in CIS patients when they were studied again after conversion to MS [139]. As these findings suggest that WM damage occurs early but progresses slowly, the information yielded by a global diffusion assessment of normal appearing brain tissue may be of limited value as a means of following disease progression, at least at disease onset. By contrast, serial diffusion MRI images in PPMS patients have detected progressive NAWM changes, which proved to be related to both lesion volume and the development of clinical disability [142]. The role of DTI metrics as predictive markers has also been highlighted in other studies that have evaluated various MS phenotypes. Although agreement on this question is not unanimous $[48,138]$, some authors have demonstrated not only that altered regional NAWM metrics are predictive of clinical impairment [46, 117], of the development of new T2 lesions and atrophy $[43,131]$, and of the future risk of MS development [143] but also that more severe diffusivity brain abnormalities in WM and GM predict higher disability $[78,144]$. DTI metrics also proved to be reliable as predictive markers of disease course when the SC was evaluated in patients with cervical relapses. Indeed, a lower RD in the lateral columns at baseline was associated with a better clinical outcome, with a greater decrease in RD being observed as patients improved clinically during followup [145].

Common guidelines are required for longitudinal DTI studies to overcome the existing discrepancies in such studies. Further regional trials designed to detect more focused MRI abnormalities, which may be used to monitor structural changes over time and, consequently, to shed light on clinical features, are warranted. Indeed, in order to be considered as an outcome measure in clinical trials, DTI parameters must be sensitive to change over time and must be highly reproducible and the sample size of the studied cohort must be appropriate to ensure the reliability of the results. The sample size needs to be planned on the basis of the statistical methods to be adopted, of the precision of the information required, and of the number of hypotheses to be tested, and in such a way as to take in account any missing values or drop-out patients. One interesting study conducted a power analysis to calculate reasonable sample sizes for longitudinal DTI studies. In brief, the authors of that study found that approximately 40 participants per arm were required for 1- to 2 -year longitudinal DTI trials, though the number could vary according to the MS phenotypes and the anatomical region being evaluated [146].

\section{Conclusions}

Diffusion abnormalities in the NAWM and NAGM have been demonstrated in all phenotypes of MS, with microstructural changes being detected in early disease stages, even in early onset MS. Nevertheless, diffusion metrics appear to differ according to the MS phenotype and within different kinds of lesions. Since MS phenotypes display different diffusivity patterns, which may be due to specific pathological substrates, diffusion measures may represent useful markers of different MS subtypes. Moreover, when diffusion measures are combined with other MRI findings, they may provide complementary information on different types of pathological damage induced by MS. Lastly, given their high sensitivity in detecting structural tissue abnormalities, DTI measures have been proposed as prognostic markers of disease course and as a means of monitoring anatomical changes over time; further studies are warranted in this field to achieve more consistent results.

\section{References}

[1] M. Filippi, "Magnetic resonance imaging findings predicting subsequent disease course in patients at presentation with clinically isolated syndromes suggestive of multiple sclerosis," Neurological Sciences, vol. 22, no. 2, pp. S49-S51, 2001.

[2] D. Le Bihan, E. Breton, and D. Lallemand, "MR imaging of intravoxel incoherent motions: application to diffusion and perfusion in neurologic disorders," Radiology, vol. 161, no. 2, pp. 401-407, 1986.

[3] R. J. Fox, "Picturing multiple sclerosis: conventional and diffusion tensor imaging," Seminars in Neurology, vol. 28, no. 4, pp. 453-466, 2008.

[4] P. J. Basser and C. Pierpaoli, "Microstructural and physiological features of tissues elucidated by quantitative-diffusion-tensor MRI," Journal of Magnetic Resonance. Series B, vol. 111, no. 3, pp. 209-219, 1996.

[5] S. K. Song, S. W. Sun, M. J. Ramsbottom, C. Chang, J. Russell, and A. H. Cross, "Dysmyelination revealed through MRI as increased radial (but unchanged axial) diffusion of water," NeuroImage, vol. 17, no. 3, pp. 1429-1436, 2002.

[6] F. Fink, J. Klein, M. Lanz et al., "Comparison of diffusion tensorbased tractography and quantified brain atrophy for analyzing demyelination and axonal loss in MS," Journal of Neuroimaging, vol. 20, no. 4, pp. 334-344, 2010.

[7] D. Goldberg-Zimring, A. U. J. Mewes, M. Maddah, and S. K. Warfield, "Diffusion tensor magnetic resonance imaging in multiple sclerosis," Journal of Neuroimaging, vol. 15, no. 4, pp. 68S-81S, 2005.

[8] C. Pierpaoli, P. Jezzard, P. J. Basser, A. Barnett, and G. Di Chiro, "Diffusion tensor MR imaging of the human brain," Radiology, vol. 201, no. 3, pp. 637-648, 1996.

[9] J. P. Mottershead, K. Schmierer, M. Clemence et al., "High field MRI correlates of myelin content and axonal density in multiple sclerosis: a post-mortem study of the spinal cord," Journal of Neurology, vol. 250, no. 11, pp. 1293-1301, 2003.

[10] J. Kolasinski, C. J. Stagg, S. A. Chance et al., "A combined post-mortem magnetic resonance imaging and quantitative histological study of multiple sclerosis pathology," Brain, vol. 135, pp. 2938-2951, 2012.

[11] K. Schmierer, C. A. M. Wheeler-Kingshott, P. A. Boulby et al., "Diffusion tensor imaging of post mortem multiple sclerosis brain," NeuroImage, vol. 35, no. 2, pp. 467-477, 2007.

[12] M. Filippi, G. Iannucci, M. Cercignani, M. A. Rocca, A. Pratesi, and G. Comi, "A quantitative study of water diffusion in multiple sclerosis lesions and normal-appearing white matter using echo-planar imaging," Archives of Neurology, vol. 57, no. 7, pp. 1017-1021, 2000.

[13] M. A. Horsfield, H. B. W. Larsson, D. K. Jones, and A. Gass, "Diffusion magnetic resonance imaging in multiple sclerosis," 
Journal of Neurology Neurosurgery and Psychiatry, vol. 64, no. 1, pp. S80-S84, 1998.

[14] D. J. Werring, C. A. Clark, G. J. Barker, A. J. Thompson, and D. H. Miller, "Diffusion tensor imaging of lesions and normalappearing white matter in multiple sclerosis," Neurology, vol. 52, no. 8, pp. 1626-1632, 1999.

[15] P. Mukherjee, J. H. Miller, J. S. Shimony et al., "Normal brain maturation during childhood: developmental trends characterized with diffusion-tensor MR imaging," Radiology, vol. 221, no. 2, pp. 349-358, 2001.

[16] P. S. Hüppi, S. E. Maier, S. Peled et al., "Microstructural development of human newborn cerebral white matter assessed in vivo by diffusion tensor magnetic resonance imaging," Pediatric Research, vol. 44, pp. 584-590, 1998.

[17] Y. Assaf and O. Pasternak, "Diffusion tensor imaging (DTI)based white matter mapping in brain research: a review," Journal of Molecular Neuroscience, vol. 34, no. 1, pp. 51-61, 2008.

[18] H. B. W. Larsson, C. Thomsen, J. Frederiksen, M. Stubgaard, and O. Henriksen, "In vivo magnetic resonance diffusion measurement in the brain of patients with multiple sclerosis," Magnetic Resonance Imaging, vol. 10, no. 1, pp. 7-12, 1992.

[19] A. G. Droogan, C. A. Clark, D. J. Werring, G. J. Barker, W. I. McDonald, and D. H. Miller, "Comparison of multiple sclerosis clinical subgroups using navigated spin echo diffusionweighted imaging," Magnetic Resonance Imaging, vol. 17, no. 5, pp. 653-661, 1999.

[20] P. Christiansen, P. Gideon, C. Thomsen, M. Stubgaard, O. Henriksen, and H. B. W. Larsson, "Increased water self-diffusion in chronic plaques and in apparently normal white matter in patients with multiple sclerosis," Acta Neurologica Scandinavica, vol. 87, no. 3, pp. 195-199, 1993.

[21] M. A. Horsfield, M. Lai, S. L. Webb et al., "Apparent diffusion coefficients in benign and secondary progressive multiple sclerosis by nuclear magnetic resonance," Magnetic Resonance in Medicine, vol. 36, no. 3, pp. 393-400, 1996.

[22] M. Cercignani, M. Inglese, E. Pagani, G. Comi, and M. Filippi, "Mean diffusivity and fractional anisotropy histograms of patients with multiple sclerosis," AJNR. American Journal of Neuroradiology, vol. 22, no. 5, pp. 952-958, 2001.

[23] M. Cercignani, G. Iannucci, M. A. Rocca, G. Comi, M. A. Horsfield, and M. Filippi, "Pathologic damage in MS assessed by diffusion-weighted and magnetization transfer MRI," Neurology, vol. 54, no. 5, pp. 1139-1144, 2000.

[24] A. O. Nusbaum, C. Y. Tang, T. C. Wei, M. S. Buchsbaum, and S. W. Atlas, "Whole-brain diffusion MR histograms differ between MS subtypes," Neurology, vol. 54, no. 7, pp. 1421-1426, 2000.

[25] S. Pajevic and C. Pierpaoli, "Color schemes to represent the orientation of anisotropic tissues from diffusion tensor data: application to white matter fiber tract mapping in the human brain," Magnetic Resonance in Medicine, vol. 42, pp. 526-540, 1999.

[26] D. K. Jones, M. R. Symms, M. Cercignani, and R. J. Howard, "The effect of filter size on VBM analyses of DT-MRI data," NeuroImage, vol. 26, no. 2, pp. 546-554, 2005.

[27] P. Preziosa, M. A. Rocca, S. Mesaros et al., "Intrinsic damage to the major white matter tracts in patients with different clinical phenotypes of multiple sclerosis: a voxelwise diffusion-tensor MR study," Radiology, vol. 260, no. 2, pp. 541-550, 2011.

[28] S. M. Smith, M. Jenkinson, H. Johansen-Berg et al., "Tractbased spatial statistics: voxelwise analysis of multi-subject diffusion data," NeuroImage, vol. 31, no. 4, pp. 1487-1505, 2006.
[29] S. Mori, B. J. Crain, V. P. Chacko, and P. C. Van Zijl, “Threedimensional tracking of axonal projections in the brain by magnetic resonance imaging," Annals of Neurology, vol. 45, pp. 265-269, 1999.

[30] M. Catani and M. Thiebaut de Schotten, "A diffusion tensor imaging tractography atlas for virtual in vivo dissections," Cortex, vol. 44, no. 8, pp. 1105-1132, 2008.

[31] P. J. Basser, S. Pajevic, C. Pierpaoli, J. Duda, and A. Aldroubi, "In vivo fiber tractography using DT-MRI data," Magnetic Resonance in Medicine, vol. 44, pp. 625-632, 2000.

[32] T. E. Conturo, N. F. Lori, T. S. Cull et al., "Tracking neuronal fiber pathways in the living human brain," Proceedings of the National Academy of Sciences of the United States of America, vol. 96, no. 18, pp. 10422-10427, 1999.

[33] S. Mori and P. C. M. Van Zijl, "Fiber tracking: principles and strategies-a technical review," NMR in Biomedicine, vol. 15, no. 7-8, pp. 468-480, 2002.

[34] R. G. Henry, J. Oh, S. J. Nelson, and D. Pelletier, "Directional diffusion in relapsing-remitting multiple sclerosis: a possible in vivo signature of Wallerian degeneration," Journal of Magnetic Resonance Imaging, vol. 18, no. 4, pp. 420-426, 2003.

[35] E. Pagani, R. Bammer, M. A. Horsfield et al., "Diffusion MR imaging in multiple sclerosis: technical aspects and challenges," AJNR. American Journal of Neuroradiology, vol. 28, no. 3, pp. 411-420, 2007.

[36] P. G. P. Nucifora, R. Verma, S. K. Lee, and E. R. Melhem, "Diffusion-tensor MR imaging and tractography: exploring brain microstructure and connectivity," Radiology, vol. 245, no. 2, pp. 367-384, 2007.

[37] T. Hendler, P. Pianka, M. Sigal et al., "Delineating gray and white matter involvement in brain lesions: three-dimensional alignment of functional magnetic resonance and diffusiontensor imaging," Journal of Neurosurgery, vol. 99, no. 6, pp. 10181027, 2003.

[38] H. Parmar, Y. Y. Sitoh, and T. T. Yeo, "Combined magnetic resonance tractography and functional magnetic resonance imaging in evaluation of brain tumors involving the motor system," Journal of Computer Assisted Tomography, vol. 28, no. 4, pp. 551-556, 2004.

[39] M. Guye, G. J. M. Parker, M. Symms et al., "Combined functional MRI and tractography to demonstrate the connectivity of the human primary motor cortex in vivo," NeuroImage, vol. 19, no. 4, pp. 1349-1360, 2003.

[40] E. Pagani, M. Filippi, M. A. Rocca, and M. A. Horsfield, "A method for obtaining tract-specific diffusion tensor MRI measurements in the presence of disease: application to patients with clinically isolated syndromes suggestive of multiple sclerosis," NeuroImage, vol. 26, no. 1, pp. 258-265, 2005.

[41] R. Bammer, M. Augustin, S. Strasser-Fuchs et al., "Magnetic resonance diffusion tensor imaging for characterizing diffuse and focal white matter abnormalities in multiple sclerosis," Magnetic Resonance in Medicine, vol. 44, pp. 583-591, 2000.

[42] O. Ciccarelli, D. J. Werring, C. A. M. Wheeler-Kingshott et al., "Investigation of MS normal-appearing brain using diffusion tensor MRI with clinical correlations," Neurology, vol. 56, no. 7, pp. 926-933, 2001.

[43] M. A. Rocca, M. Cercignani, G. Iannucci, G. Comi, and M. Filippi, "Weekly diffusion-weighted imaging of normalappearing white matter in MS," Neurology, vol. 55, no. 6, pp. 882-884, 2000. 
[44] A. C. Guo, V. L. Jewells, and J. M. Provenzale, "Analysis of normal-appearing white Matter in multiple sclerosis: comparison of diffusion tensor MR imaging and magnetization transfer imaging," AJNR. American Journal of Neuroradiology, vol. 22, no. 10, pp. 1893-1900, 2001.

[45] N. Evangelou, D. Konz, M. M. Esiri, S. Smith, J. Palace, and P. M. Matthews, "Regional axonal loss in the corpus callosum correlates with cerebral white matter lesion volume and distribution in multiple sclerosis," Brain, vol. 123, no. 9, pp. 1845-1849, 2000.

[46] D. J. Werring, D. Brassat, A. G. Droogan et al., "The pathogenesis of lesions and normal-appearing white matter changes in multiple sclerosis. A serial diffusion MRI study," Brain, vol. 123, no. 8, pp. 1667-1676, 2000.

[47] M. S. Vishwas, B. C. Healy, R. Pienaar, M. P. Gorman, P. E. Grant, and T. Chitnis, "Diffusion tensor analysis of pediatric multiple sclerosis and clinically isolated syndromes," AJNR. American Journal of Neuroradiology, vol. 34, no. 2, pp. 417-423, 2013.

[48] A. Gallo, M. Rovaris, R. Riva et al., "Diffusion-tensor magnetic resonance imaging detects normal-appearing white matter damage unrelated to short-term disease activity in patients at the earliest clinical stage of multiple sclerosis," Archives of Neurology, vol. 62, no. 5, pp. 803-808, 2005.

[49] R. G. Henry, M. Shieh, B. Amirbekian, S. Chung, D. T. Okuda, and D. Pelletier, "Connecting white matter injury and thalamic atrophy in clinically isolated syndromes," Journal of the Neurological Sciences, vol. 282, no. 1-2, pp. 61-66, 2009.

[50] J. Tillema, J. Leach, and I. Pirko, "Non-lesional white matter changes in pediatric multiple sclerosis and monophasic demyelinating disorders," Multiple Sclerosis, vol. 18, pp. 17541759, 2012.

[51] P. Tortorella, M. A. Rocca, D. M. Mezzapesa et al., "MRI quantification of gray and white matter damage in patients with early-onset multiple sclerosis," Journal of Neurology, vol. 253, no. 7, pp. 903-907, 2006.

[52] M. S. Vishwas, T. Chitnis, R. Pienaar, B. C. Healy, and P. E. Grant, "Tract-based analysis of callosal, projection, and association pathways in pediatric patients with multiple sclerosis: a preliminary study," AJNR. American Journal of Neuroradiology, vol. 31, no. 1, pp. 121-128, 2010.

[53] E. Raz, M. Cercignani, E. Sbardella et al., "Clinically isolated syndrome suggestive of multiple sclerosis: voxelwise regional investigation of white and gray matter," Radiology, vol. 254, no. 1, pp. 227-234, 2010.

[54] A. Pulizzi, M. Rovaris, E. Judica et al., "Determinants of disability in multiple sclerosis at various disease stages: a multiparametric magnetic resonance study," Archives of Neurology, vol. 64, no. 8, pp. 1163-1168, 2007.

[55] A. Bethune, V. Tipu, J. G. Sled et al., "Diffusion tensor imaging and cognitive speed in children with multiple sclerosis," Journal of the Neurological Sciences, vol. 309, no. 1-2, pp. 68-74, 2011.

[56] T. Kuhlmann, G. Lingfeld, A. Bitsch, J. Schuchardt, and W. Brück, "Acute axonal damage in multiple sclerosis is most extensive in early disease stages and decreases over time," Brain, vol. 125, no. 10, pp. 2202-2212, 2002.

[57] C. S. Yu, F. C. Lin, Y. Liu, Y. Duan, H. Lei, and K. C. Li, "Histogram analysis of diffusion measures in clinically isolated syndromes and relapsing-remitting multiple sclerosis," European Journal of Radiology, vol. 68, no. 2, pp. 328-334, 2008.

[58] B. Spanò, M. Cercignani, B. Basile et al., "Multiparametric MR investigation of the motor pyramidal system in patients with ?truly benign? multiple sclerosis," Multiple Sclerosis, vol. 16, no. 2, pp. 178-188, 2010.
[59] A. Ceccarelli, M. A. Rocca, E. Pagani et al., "The topographical distribution of tissue injury in benign MS: a 3T multiparametric MRI study," NeuroImage, vol. 39, no. 4, pp. 1499-1509, 2008.

[60] C. M. Griffin, D. T. Chard, O. Ciccarelli et al., "Diffusion tensor imaging in early relapsing — remitting multiple sclerosis," Multiple Sclerosis, vol. 7, no. 5, pp. 290-297, 2001.

[61] T. J. Braley, Y. H. Lee, S. Mohan, B. M. Segal, S. Berini, and A. Srinivasan, "Differences in diffusion tensor imaging-derived metrics in the corpus callosum of patients with multiple sclerosis without and with gadolinium-enhancing cerebral lesions," Journal of Computer Assisted Tomography, vol. 36, no. 4, pp. 410415, 2012.

[62] A. C. Scanderbeg, F. Tomaiuolo, U. Sabatini, U. Nocentini, M. G. Grasso, and C. Caltagirone, "Demyelinating plaques in relapsing-remitting and secondary-progressive multiple sclerosis: assessment with diffusion MR imaging," AJNR. American Journal of Neuroradiology, vol. 21, no. 5, pp. 862-868, 2000.

[63] P. E. Sijens, R. Irwan, J. H. Potze, J. P. Mostert, J. De Keyser, and M. Oudkerk, "Analysis of the human brain in primary progressive multiple sclerosis with mapping of the spatial distributions using $1 \mathrm{H}$ MR spectroscopy and diffusion tensor imaging," European Radiology, vol. 15, no. 8, pp. 1686-1693, 2005.

[64] M. Rovaris, M. Bozzali, G. Iannucci et al., "Assessment of normal-appearing white and gray matter in patients with primary progressive multiple sclerosis: a diffusion-tensor magnetic resonance imaging study," Archives of Neurology, vol. 59, no. 9, pp. 1406-1412, 2002.

[65] A. Cifelli, M. Arridge, P. Jezzard, M. M. Esiri, J. Palace, and P. M. Matthews, "Thalamic neurodegeneration in multiple sclerosis," Annals of Neurology, vol. 52, no. 5, pp. 650-653, 2002.

[66] A. Kutzelnigg and H. Lassmann, "Cortical lesions and brain atrophy in MS," Journal of the Neurological Sciences, vol. 233, no. 1-2, pp. 55-59, 2005.

[67] K. M. Hasan, C. Halphen, A. Kamali, F. M. Nelson, J. S. Wolinsky, and P. A. Narayana, "Caudate nuclei volume, diffusion tensor metrics, and T2 relaxation in healthy adults and relapsing-remitting multiple sclerosis patients: implications for understanding gray matter degeneration," Journal of Magnetic Resonance Imaging, vol. 29, no. 1, pp. 70-77, 2009.

[68] A. J. Fabiano, J. Sharma, B. Weinstock-Guttman et al., "Thalamic involvement in multiple sclerosis: a diffusion-weighted magnetic resonance imaging study," Journal of Neuroimaging, vol. 13, no. 4, pp. 307-314, 2003.

[69] A. Ceccarelli, M. A. Rocca, P. Valsasina et al., "A multiparametric evaluation of regional brain damage in patients with primary progressive multiple sclerosis," Human Brain Mapping, vol. 30, no. 9, pp. 3009-3019, 2009.

[70] F. Zhou, C. S. Zee, H. Gong, M. Shiroishi, and J. Li, "Differential changes in deep and cortical gray matters of patients with multiple sclerosis: a quantitative magnetic resonance imaging study," Journal of Computer Assisted Tomography, vol. 34, no. 3, pp. 431-436, 2010.

[71] M. Bozzali, M. Cercignani, M. P. Sormani, G. Comi, and M. Filippi, "Quantification of brain gray matter damage in different MS phenotypes by use of diffusion tensor MR imaging," AJNR. American Journal of Neuroradiology, vol. 23, no. 6, pp. 985-988, 2002.

[72] H. Vrenken, P. J. W. Pouwels, J. J. G. Geurts et al., "Altered diffusion tensor in multiple sclerosis normal-appearing brain tissue: cortical diffusion changes seem related to clinical deterioration," 
Journal of Magnetic Resonance Imaging, vol. 23, no. 5, pp. 628636, 2006.

[73] C. Oreja-Guevara, M. Rovaris, G. Iannucci et al., "Progressive gray matter damage in patients with relapsing-remitting multiple sclerosis: a longitudinal diffusion tensor magnetic resonance imaging study," Archives of Neurology, vol. 62, no. 4, pp. 578-584, 2005.

[74] M. Rovaris, G. Riccitelli, E. Judica et al., "Cognitive impairment and structural brain damage in benign multiple sclerosis," Neurology, vol. 71, no. 19, pp. 1521-1526, 2008.

[75] A. Ceccarelli, M. Filippi, M. Neema et al., "T2 hypointensity in the deep gray matter of patients with benign multiple sclerosis," Multiple Sclerosis, vol. 15, no. 6, pp. 678-686, 2009.

[76] A. Ceccarelli, M. A. Rocca, A. Falini et al., "Normal-appearing white and grey matter damage in MS: a volumetric and diffusion tensor MRI study at 3.0 Tesla," Journal of Neurology, vol. 254, no. 4, pp. 513-518, 2007.

[77] F. Tovar-Moll, I. E. Evangelou, A. W. Chiu et al., "Thalamic involvement and its impact on clinical disability in patients with multiple sclerosis: a diffusion tensor imaging study at 3T," AJNR. American Journal of Neuroradiology, vol. 30, no. 7, pp. 13801386, 2009.

[78] M. Calabrese, F. Rinaldi, D. Seppi et al., "Cortical diffusiontensor imaging abnormalities in multiple sclerosis: a 3-year longitudinal study," Radiology, vol. 261, no. 3, pp. 891-898, 2011.

[79] L. V. Zollinger, T. H. Kim, K. Hill, E. K. Jeong, and J. W. Rose, "Using diffusion tensor imaging and immunofluorescent assay to evaluate the pathology of multiple sclerosis," Journal of Magnetic Resonance Imaging, vol. 33, no. 3, pp. 557-564, 2011.

[80] E. C. Klawiter, R. E. Schmidt, K. Trinkaus et al., "Radial diffusivity predicts demyelination in ex vivo multiple sclerosis spinal cords," NeuroImage, vol. 55, no. 4, pp. 1454-1460, 2011.

[81] B. Benedetti, M. A. Rocca, M. Rovaris et al., "A diffusion tensor MRI study of cervical cord damage in benign and secondary progressive multiple sclerosis patients," Journal of Neurology, Neurosurgery and Psychiatry, vol. 81, no. 1, pp. 26-30, 2010.

[82] D. M. Mezzapesa, M. A. Rocca, A. Falini et al., "A preliminary diffusion tensor and magnetization transfer magnetic resonance imaging study of early-onset multiple sclerosis," Archives of Neurology, vol. 61, no. 3, pp. 366-368, 2004.

[83] L. C. H. Cruz Jr., R. C. Domingues, and E. L. Gasparetto, "Diffusion tensor imaging of the cervical spinal cord of patients with relapsing-remising multiple sclerosis," Arquivos de NeuroPsiquiatria, vol. 67, no. 2 B, pp. 391-395, 2009.

[84] Y. Ohgiya, M. Oka, A. Hiwatashi et al., "Diffusion tensor MR imaging of the cervical spinal cord in patients with multiple sclerosis," European Radiology, vol. 17, no. 10, pp. 2499-2504, 2007.

[85] S. M. Hesseltine, M. Law, J. Babb et al., "Diffusion tensor imaging in multiple sclerosis: assessment of regional differences in the axial plane within normal-appearing cervical spinal cord," AJNR. American Journal of Neuroradiology, vol. 27, no. 6, pp. 1189-1193, 2006.

[86] P. Valsasina, M. A. Rocca, F. Agosta et al., "Mean diffusivity and fractional anisotropy histogram analysis of the cervical cord in MS patients," NeuroImage, vol. 26, no. 3, pp. 822-828, 2005.

[87] W. Van Hecke, G. Nagels, G. Emonds et al., "A diffusion tensor imaging group study of the spinal cord in multiple sclerosis patients with and without T2 spinal cord lesions," Journal of Magnetic Resonance Imaging, vol. 30, no. 1, pp. 25-34, 2009.
[88] F. Agosta, B. Benedetti, M. A. Rocca et al., "Quantification of cervical cord pathology in primary progressive MS using diffusion tensor MRI," Neurology, vol. 64, no. 4, pp. 631-635, 2005.

[89] S. Roychowdhury, J. A. Maldjian, and R. I. Grossman, "Multiple sclerosis: comparison of trace apparent diffusion coefficients with $\mathrm{mr}$ enhancement pattern of lesions," AJNR. American Journal of Neuroradiology, vol. 21, no. 5, pp. 869-874, 2000.

[90] M. A. Rocca, D. M. Mezzapesa, A. Falini et al., "Evidence for axonal pathology and adaptive cortical reorganization in patients at presentation with clinically isolated syndromes suggestive of multiple sclerosis," NeuroImage, vol. 18, no. 4, pp. 847-855, 2003.

[91] C. H. Sotak, "The role of diffusion tensor imaging in the evaluation of ischemic brain-a review," NMR in Biomedicine, vol. 15, no. 7-8, pp. 561-569, 2002.

[92] F. Zelaya, N. Flood, J. B. Chalk et al., "An evaluation of the time dependence of the anisotropy of the water diffusion tensor in acute human ischemia," Magnetic Resonance Imaging, vol. 17, no. 3, pp. 331-348, 1999.

[93] K. E. Balashov and E. Lindzen, "Acute demyelinating lesions with restricted diffusion in multiple sclerosis," Multiple Sclerosis, vol. 18, pp. 1745-1753, 2012.

[94] R. T. Naismith, J. Xu, N. T. Tutlam, K. Trinkaus, A. H. Cross, and S. K. Song, "Radial diffusivity in remote optic neuritis discriminates visual outcomes," Neurology, vol. 74, no. 21, pp. 1702-1710, 2010.

[95] D. Kidd, F. Barkhof, R. McConnell, P. R. Algra, I. V. Allen, and T. Revesz, "Cortical lesions in multiple sclerosis," Brain, vol. 122, no. 1, pp. 17-26, 1999.

[96] A. H. Poonawalla, K. M. Hasan, R. K. Gupta et al., "Diffusiontensor MR imaging of cortical lesions in multiple sclerosis: initial findings," Radiology, vol. 246, no. 3, pp. 880-886, 2008.

[97] B. D. Trapp, J. R. Wujek, G. A. Criste et al., "Evidence for synaptic stripping by cortical microglia," Glia, vol. 55, no. 4, pp. 360-368, 2007.

[98] F. Barkhof, “The clinico-radiological paradox in multiple sclerosis revisited," Current Opinion in Neurology, vol. 15, no. 3, pp. 239-245, 2002.

[99] M. Rovaris, A. Gallo, P. Valsasina et al., "Short-term accrual of gray matter pathology in patients with progressive multiple sclerosis: an in vivo study using diffusion tensor MRI," NeuroImage, vol. 24, no. 4, pp. 1139-1146, 2005.

[100] J. F. Kurtzke, "Rating neurologic impairment in multiple sclerosis: an expanded disability status scale (EDSS)," Neurology, vol. 33, no. 11, pp. 1444-1452, 1983.

[101] Y. Liu, Y. Duan, Y. He et al., "Whole brain white matter changes revealed by multiple diffusion metrics in multiple sclerosis: a TBSS study," European Journal of Radiology, vol. 81, no. 10, pp. 2826-2832, 2012.

[102] A. Giorgio and N. De Stefano, "Cognition in multiple sclerosis: relevance of lesions, brain atrophy and proton MR spectroscopy," Neurological Sciences, vol. 31, no. 2, pp. S245-S248, 2010.

[103] M. Onu, A. Roceanu, U. Sboto-Frankenstein et al., "Diffusion abnormality maps in demyelinating disease: correlations with clinical scores," European Journal of Radiology, vol. 81, pp. e386e391, 2012.

[104] O. Ciccarelli, C. A. Wheeler-Kingshott, M. A. McLean et al., "Spinal cord spectroscopy and diffusion-based tractography to assess acute disability in multiple sclerosis," Brain, vol. 130, no. 8, pp. 2220-2231, 2007. 
[105] D. S. Reich, S. A. Smith, K. M. Zackowski et al., "Multiparametric magnetic resonance imaging analysis of the corticospinal tract in multiple sclerosis," NeuroImage, vol. 38, no. 2, pp. 271279, 2007.

[106] F. Lin, C. Yu, T. Jiang, K. Li, and P. Chan, "Diffusion tensor tractography-based group mapping of the pyramidal tract in relapsing-remitting multiple sclerosis patients," AJNR. American Journal of Neuroradiology, vol. 28, no. 2, pp. 278-282, 2007.

[107] B. Hu, B. Ye, Y. Yang et al., "Quantitative diffusion tensor deterministic and probabilistic fiber tractography in relapsingremitting multiple sclerosis," European Journal of Radiology, vol. 79, no. 1, pp. 101-107, 2011.

[108] M. Rovaris, G. Iannucci, M. Falautano et al., "Cognitive dysfunction in patients with mildly disabling relapsing-remitting multiple sclerosis: an exploratory study with diffusion tensor MR imaging," Journal of the Neurological Sciences, vol. 195, no. 2, pp. 103-109, 2002.

[109] J. Oh, R. G. Henry, C. Genain, S. J. Nelson, and D. Pelletier, "Mechanisms of normal appearing corpus callosum injury related to pericallosal $\mathrm{T} 1$ lesions in multiple sclerosis using directional diffusion tensor and 1H MRS imaging," Journal of Neurology, Neurosurgery and Psychiatry, vol. 75, no. 9, pp. 12811286, 2004.

[110] S. J. Hickman, C. A. M. Wheeler-Kingshott, S. J. Jones et al., "Optic nerve diffusion measurement from diffusion-weighted imaging in optic neuritis," AJNR. American Journal of Neuroradiology, vol. 26, no. 4, pp. 951-956, 2005.

[111] S. A. Trip, C. Wheeler-Kingshott, S. J. Jones et al., "Optic nerve diffusion tensor imaging in optic neuritis," NeuroImage, vol. 30, no. 2, pp. 498-505, 2006.

[112] S. C. Kolbe, M. Marriott, Av. Walt et al., "Diffusion tensor imaging correlates of visual impairment in multiple sclerosis and chronic optic neuritis," Investigative Ophthalmology \& Visual Science, vol. 53, no. 2, pp. 825-832, 2012.

[113] E. M. Frohman, M. G. Dwyer, T. Frohman et al., "Relationship of optic nerve and brain conventional and non-conventional MRI measures and retinal nerve fiber layer thickness, as assessed by OCT and GDx: a pilot study," Journal of the Neurological Sciences, vol. 282, no. 1-2, pp. 96-105, 2009.

[114] M. Wilson, C. R. Tench, P. S. Morgan, and L. D. Blumhardt, "Pyramidal tract mapping by diffusion tensor magnetic resonance imaging in multiple sclerosis: improving correlations with disability," Journal of Neurology Neurosurgery and Psychiatry, vol. 74, no. 2, pp. 203-207, 2003.

[115] X. Lin, C. R. Tench, P. S. Morgan, G. Niepel, and C. S. Constantinescu, "'Importance sampling' in MS: use of diffusion tensor tractography to quantify pathology related to specific impairment," Journal of the Neurological Sciences, vol. 237, no. 1-2, pp. 13-19, 2005.

[116] T. Sigal, M. Shmuel, D. Mark, H. Gil, and A. Anat, "Diffusion tensor imaging of corpus callosum integrity in multiple sclerosis: correlation with disease variables," Journal of Neuroimaging, vol. 22, pp. 33-37, 2012.

[117] K. C. Kern, J. Sarcona, M. Montag, B. S. Giesser, and N. L. Sicotte, "Corpus callosal diffusivity predicts motor impairment in relapsing-remitting multiple sclerosis: a TBSS and tractography study," NeuroImage, vol. 55, no. 3, pp. 1169-1177, 2011.

[118] V. M. Anderson, C. A. M. Wheeler-Kingshott, K. Abdel-Aziz et al., "A comprehensive assessment of cerebellar damage in multiple sclerosis using diffusion tractography and volumetric analysis," Multiple Sclerosis, vol. 17, no. 9, pp. 1079-1087, 2011.
[119] L. Prosperini, A. Kouleridou, N. Petsas et al., "The relationship between infratentorial lesions, balance deficit and accidental falls in multiple sclerosis," Journal of the Neurological Sciences, vol. 304, no. 1-2, pp. 55-60, 2011.

[120] R. A. Dineen, J. Vilisaar, J. Hlinka et al., "Disconnection as a mechanism for cognitive dysfunction in multiple sclerosis," Brain, vol. 132, no. 1, pp. 239-249, 2009.

[121] H. J. Yu, C. Christodoulou, V. Bhise et al., "Multiple white matter tract abnormalities underlie cognitive impairment in RRMS," Neuroimage, vol. 59, no. 4, pp. 3713-3722, 2012.

[122] L. Bonzano, A. Tacchino, L. Roccatagliata, M. P. Sormani, G. L. Mancardi, and M. Bove, "Impairment in explicit visuomotor sequence learning is related to loss of microstructural integrity of the corpus callosum in multiple sclerosis patients with minimal disability," NeuroImage, vol. 57, no. 2, pp. 495-501, 2011.

[123] S. Llufriu, Y. Blanco, E. Martinez-Heras et al., "Influence of corpus callosum damage on cognition and physical disability in multiple sclerosis: a multimodal study," PLoS ONE, vol. 7, no. 5, Article ID e37167, 2012.

[124] B. Audoin, M. Guye, F. Reuter et al., "Structure of WM bundles constituting the working memory system in early multiple sclerosis: a quantitative DTI tractography study," NeuroImage, vol. 36, no. 4, pp. 1324-1330, 2007.

[125] S. D. Roosendaal, J. J. G. Geurts, H. Vrenken et al., "Regional DTI differences in multiple sclerosis patients," NeuroImage, vol. 44, no. 4, pp. 1397-1403, 2009.

[126] X. Lin, C. R. Tench, P. S. Morgan, and C. S. Constantinescu, "Use of combined conventional and quantitative MRI to quantify pathology related to cognitive impairment in multiple sclerosis," Journal of Neurology, Neurosurgery and Psychiatry, vol. 79, no. 4, pp. 437-441, 2008.

[127] M. Roca, T. Torralva, F. Meli et al., "Cognitive deficits in multiple sclerosis correlate with changes in fronto-subcortical tracts," Multiple Sclerosis, vol. 14, no. 3, pp. 364-369, 2008.

[128] C. Till, A. Deotto, V. Tipu et al., "White matter integrity and math performance in pediatric multiple sclerosis: a diffusion tensor imaging study," Neuroreport, vol. 22, no. 18, pp. 10051009, 2011.

[129] S. Cader, H. Johansen-Berg, M. Wylezinska et al., "Discordant white matter $\mathrm{N}$-acetylasparate and diffusion MRI measures suggest that chronic metabolic dysfunction contributes to axonal pathology in multiple sclerosis," NeuroImage, vol. 36, no. 1, pp. 19-27, 2007.

[130] S. Hannoun, F. Durand-Dubief, C. Confavreux et al., "Diffusion tensor-MRI evidence for extra-axonal neuronal degeneration in caudate and thalamic nuclei of patients with multiple sclerosis," AJNR. American Journal of Neuroradiology, vol. 33, no. 7, pp. 1363-1368, 2012.

[131] J. P. Ranjeva, J. Pelletier, S. Confort-Gouny et al., "MRI/MRS of corpus callosum in patients with clinically isolated syndrome suggestive of multiple sclerosis," Multiple Sclerosis, vol. 9, no. 6, pp. 554-565, 2003.

[132] M. C. G. Otaduy, D. Callegaro, L. A. Bacheschi, and C. C. Leite, "Correlation of magnetization transfer and diffusion magnetic resonance imaging in multiple sclerosis," Multiple Sclerosis, vol. 12, no. 6, pp. 754-759, 2006.

[133] G. Iannucci, M. Rovaris, L. Giacomotti, G. Comi, and M. Filippi, "Correlation of multiple sclerosis measures derived from T2-weighted, T1-weighted, magnetization transfer, and diffusion tensor MR imaging," AJNR. American Journal of Neuroradiology, vol. 22, no. 8, pp. 1462-1467, 2001. 
[134] M. A. Rocca, A. Falini, B. Colombo, G. Scotti, G. Comi, and M. Filippi, "Adaptive functional changes in the cerebral cortex of patients with nondisabling multiple sclerosis correlate with the extent of brain structural damage," Annals of Neurology, vol. 51, no. 3, pp. 330-339, 2002.

[135] D. J. Werring, C. A. Clark, G. J. Parker, D. H. Miller, A. J. Thompson, and G. J. Barker, "A direct demonstration of both structure and function in the visual system: combining diffusion tensor imaging with functional magnetic resonance imaging," Neuroimage, vol. 9, no. 3, pp. 352-361, 1999.

[136] D. Lenzi, A. Conte, C. Mainero et al., "Effect of corpus callosum damage on ipsilateral motor activation in patients with multiple sclerosis: a functional and anatomical study," Human Brain Mapping, vol. 28, no. 7, pp. 636-644, 2007.

[137] D. J. Hawellek, J. F. Hipp, C. M. Lewis, M. Corbetta, and A. K. Engel, "Increased functional connectivity indicates the severity of cognitive impairment in multiple sclerosis," Proceedings of the National Academy of Sciences of the United States of America, vol. 108, pp. 19066-19071, 2011.

[138] M. Rovaris, E. Judica, A. Ceccarelli et al., "A 3-year diffusion tensor MRI study of grey matter damage progression during the earliest clinical stage of MS," Journal of Neurology, vol. 255, no. 8, pp. 1209-1214, 2008.

[139] E. Raz, M. Cercignani, E. Sbardella et al., "Gray- and whitematter changes 1 year after first clinical episode of multiple sclerosis: MR imaging," Radiology, vol. 257, no. 2, pp. 448-454, 2010.

[140] F. Agosta, M. Absinta, M. P. Sormani et al., "In vivo assessment of cervical cord damage in MS patients: a longitudinal diffusion tensor MRI study," Brain, vol. 130, no. 8, pp. 2211-2219, 2007.

[141] W. Rashid, A. Hadjiprocopis, G. Davies et al., "Longitudinal evaluation of clinically early relapsing-remitting multiple sclerosis with diffusion tensor imaging," Journal of Neurology, vol. 255, no. 3, pp. 390-397, 2008.

[142] K. Schmierer, D. R. Altmann, N. Kassim et al., "Progressive change in primary progressive multiple sclerosis normalappearing white matter: a serial diffusion magnetic resonance imaging study," Multiple Sclerosis, vol. 10, no. 2, pp. 182-187, 2004.

[143] M. Bester, C. Heesen, S. Schippling et al., "Early anisotropy changes in the corpus callosum of patients with optic neuritis," Neuroradiology, vol. 50, no. 7, pp. 549-557, 2008.

[144] M. Rovaris, E. Judica, A. Gallo et al., "Grey matter damage predicts the evolution of primary progressive multiple sclerosis at 5 years," Brain, vol. 129, no. 10, pp. 2628-2634, 2006.

[145] P. Freund, C. Wheeler-Kingshott, J. Jackson, D. Miller, A. Thompson, and O. Ciccarelli, "Recovery after spinal cord relapse in multiple sclerosis is predicted by radial diffusivity," Multiple Sclerosis, vol. 16, no. 10, pp. 1193-1202, 2010.

[146] D. M. Harrison, B. S. Caffo, N. Shiee et al., "Longitudinal changes in diffusion tensor-based quantitative MRI in multiple sclerosis," Neurology, vol. 76, no. 2, pp. 179-186, 2011. 


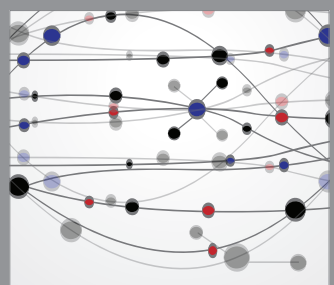

The Scientific World Journal
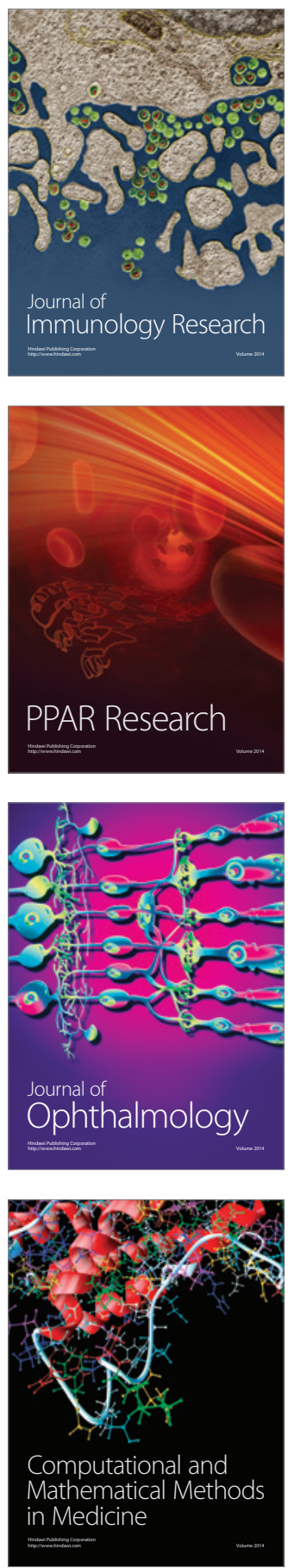

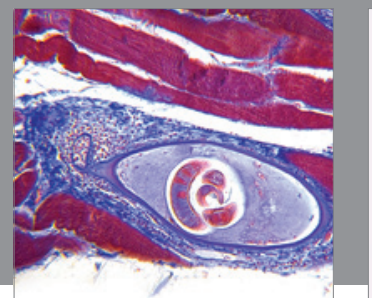

Gastroenterology

Research and Practice
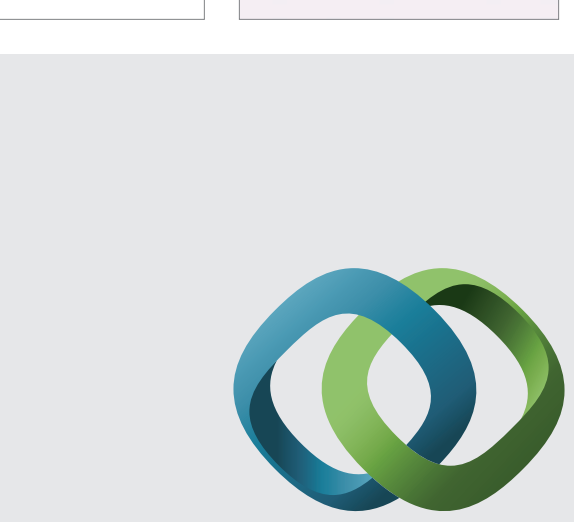

\section{Hindawi}

Submit your manuscripts at

http://www.hindawi.com
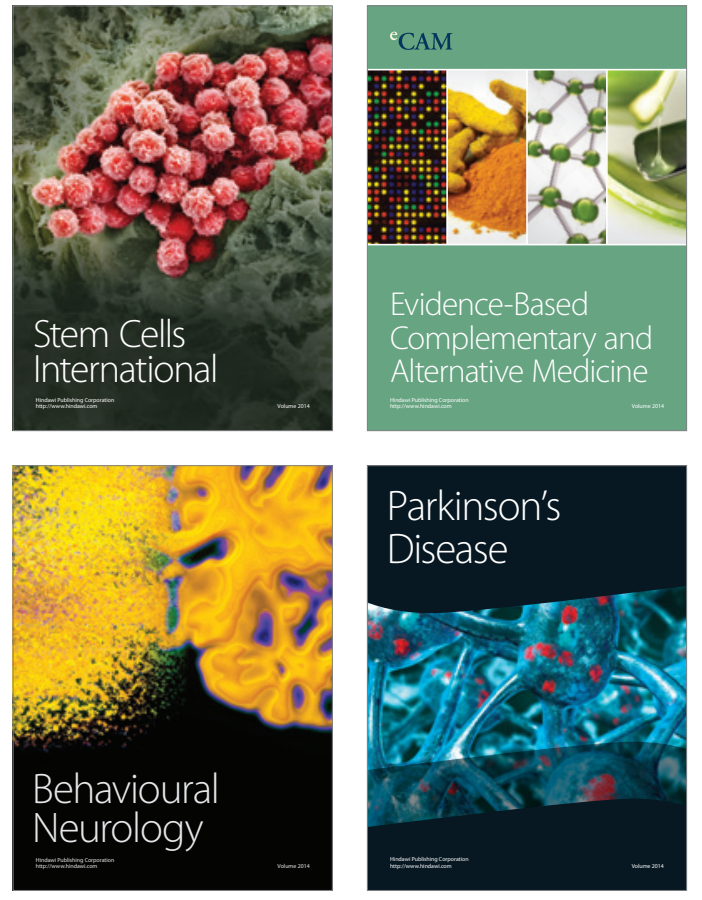
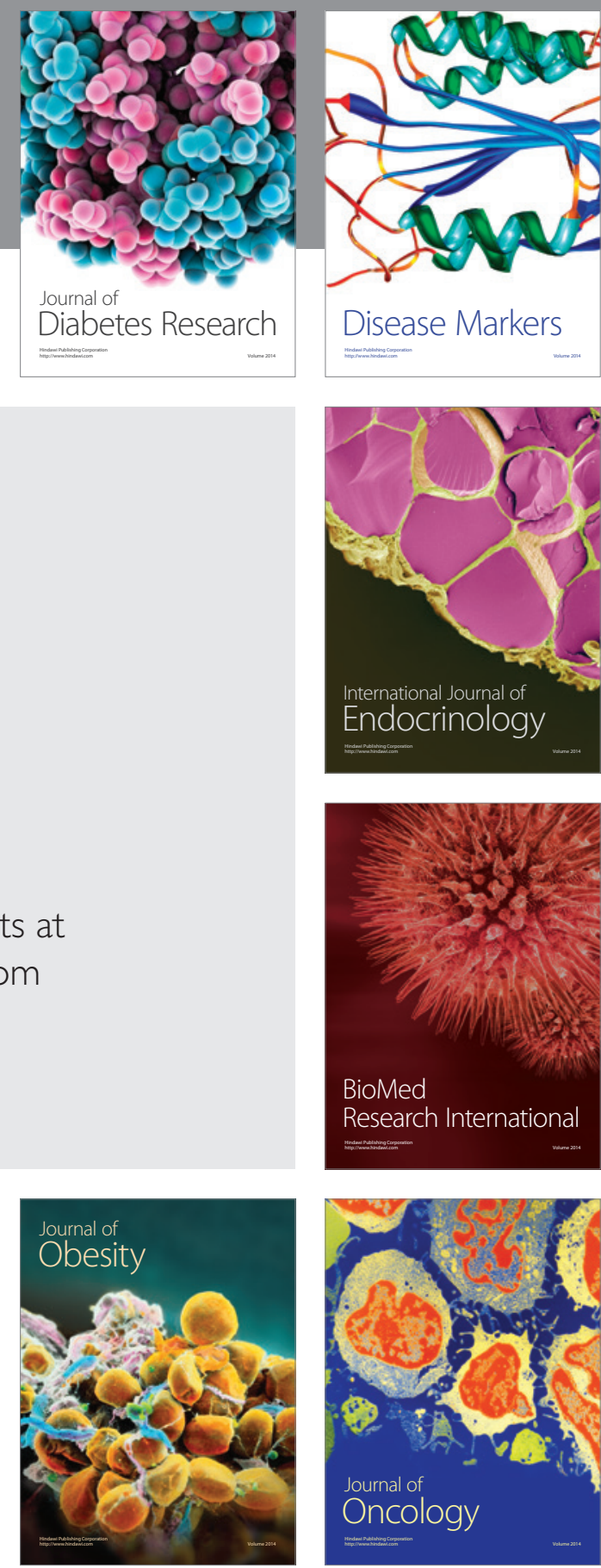

Disease Markers
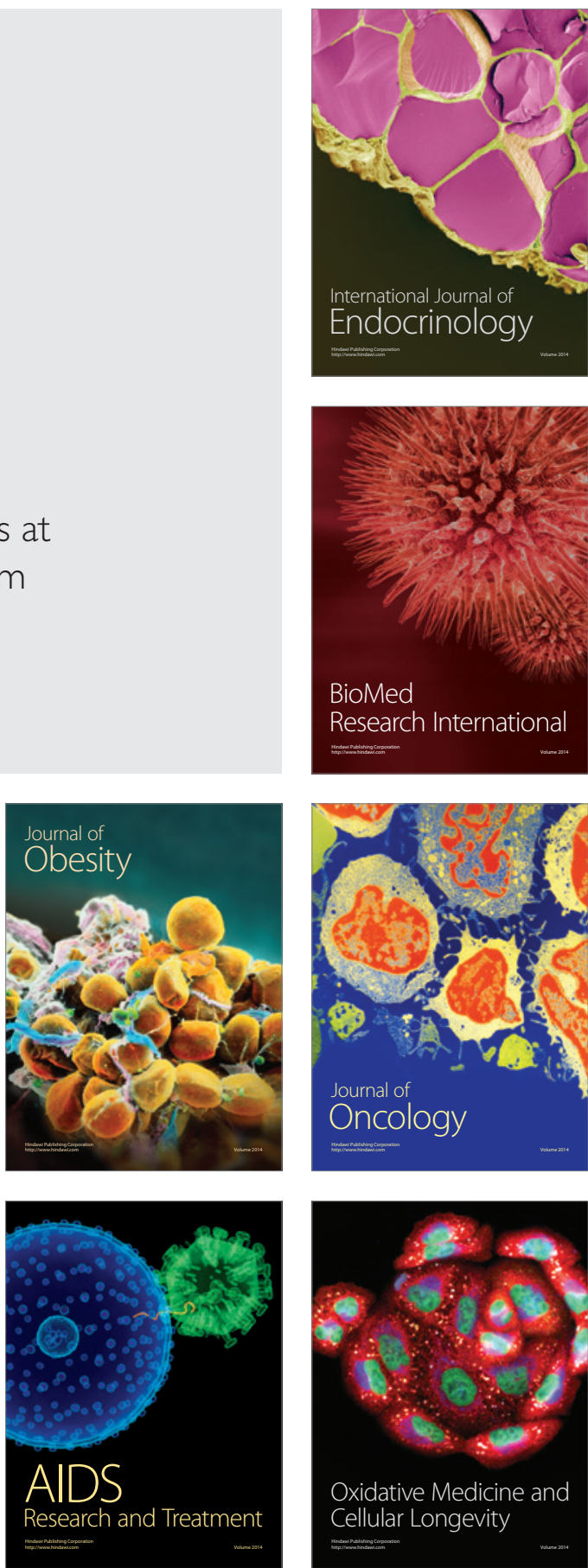